Bull. Korean Math. Soc. 50 (2013), No. 6, pp. 2071-2077

http://dx.doi.org/10.4134/BKMS.2013.50.6.2071

\title{
CONICS ON A GENERAL HYPERSURFACE IN COMPLEX PROJECTIVE SPACES
}

\author{
DONGSOO SHIN
}

\begin{abstract}
In this paper we consider the existence of smooth conics on a general hypersurface of degree $d$ in $\mathbb{P}^{n}$.
\end{abstract}

\section{Introduction}

Let $X$ be a general hypersurface of degree $d$ in a complex projective space $\mathbb{P}^{n}$. Recall that $R_{e}(X)$ is the open subscheme of the Hilbert scheme Hilb ${ }^{e t+1}(X)$ which parameterizes smooth rational curves of degree $e$ lying on $X$. Recently J. Harris, M. Roth, and J. Starr obtained the following general result for $d<\frac{n+1}{2}$ :

Theorem (Harris-Roth-Starr [2]). If $n>2$ and $d<\frac{n+1}{2}$, then $R_{e}(X)$ is an integral, local complete intersection scheme of dimension $(n+1-d) e+(n-4)$ for every $e \geq 1$.

However the upper bound of $d<\frac{n+1}{2}$ in the above theorem is not optimal for a certain degree $e$. For instance, we have a sharp bound for the degree $d$ in case of $e=1$ :

Theorem (Barth-Van de Ven [1]). If $d>2 n-3$, then $R_{1}(X)$ is empty. If $d \leq 2 n-3$, then $R_{1}(X)$ is smooth of dimension $2 n-3-d$.

In this paper we will investigate the nonemptyness and smoothness of $R_{2}(X)$.

Theorem 1.1. Let $X$ be a general hypersurface of degree $d$ in $\mathbb{P}^{n}$.

(a) If $2 d>3 n-2$, then $R_{2}(X)$ is empty.

(b) If $2 d \leq 3 n-2$, then $R_{2}(X)$ is smooth and of dimension $3 n-2 d-2$.

\section{Proof}

This section is devoted to the proof of Theorem 1.1.

Received January 3, 2013; Revised March 20, 2013.

2010 Mathematics Subject Classification. 14H10.

Key words and phrases. conic, general hypersurface, Hilbert scheme. 
Let $\mathbb{G}(2, n)$ be the Grassmannian of 2 -dimensional subspaces in $\mathbb{P}^{n}$ and $U \rightarrow$ $\mathbb{G}(2, n)$ the universal bundle. Since every conic is contained in a unique $\mathbb{P}^{2}$ in $\mathbb{P}^{n}$, it follows that

$$
\operatorname{Hilb}^{2 t+1}\left(\mathbb{P}^{n}\right) \cong \mathbb{P}\left(\operatorname{sym}^{2} U^{*}\right) .
$$

Hence $R_{2}\left(\mathbb{P}^{n}\right)$ is open in $\mathbb{P}\left(\operatorname{sym}^{2} U^{*}\right)$. Let $H_{d}$ be the parameter space of hypersurfaces of degree $d$ in $\mathbb{P}^{n}$, i.e., $H_{d}=\mathbb{P}^{N}, N=\left(\begin{array}{c}n+d \\ d\end{array}\right)-1$. Define the incidence scheme

$$
J_{d}=\left\{(C, F) \in R_{2}\left(\mathbb{P}^{n}\right) \times H_{d}: C \subset F\right\}
$$

and consider two projections $p_{R}: J_{d} \rightarrow R_{2}\left(\mathbb{P}^{n}\right)$ and $p_{H}: J_{d} \rightarrow H_{d}$. Then we have

$$
R_{2}(X) \cong p_{H}^{-1}(X) .
$$

To prove Theorem 1.1, we modify the proof in Kollár [5, Theorem 4.3] which considers lines on a general hypersurface. The idea is a comparison of the dimensions of $J_{d}$ and the parameter space $H_{d}$. A similar technique is used in Katz [4] which shows that there are 609250 conics on a general quintic threefold.

We begin with the following lemma.

Lemma 2.1. The incidence scheme $J_{d}$ is irreducible, nonsingular, and of codimension $2 d+1$ in $R_{2}\left(\mathbb{P}^{n}\right) \times H_{d}$.

Proof. Since we have

$$
\operatorname{Hilb}^{2 t+1}\left(\mathbb{P}^{n}\right) \cong \mathbb{P}\left(\operatorname{sym}^{2} U^{*}\right),
$$

it follows that $\operatorname{Hilb}^{2 t+1}\left(\mathbb{P}^{n}\right)$ is irreducible because $\mathbb{P}\left(\operatorname{sym}^{2} U^{*}\right)$ is a vector bundle on the irreducible variety $\mathbb{G}(2, n)$. Therefore $R_{2}\left(\mathbb{P}^{n}\right)$ is irreducible because $R_{2}\left(\mathbb{P}^{n}\right)$ is open in $\mathbb{P}\left(\operatorname{sym}^{2} U^{*}\right)$.

Note that

$$
p_{R}^{-1}(C)=\operatorname{ker}\left(\alpha: H^{0}\left(\mathbb{P}^{n}, \mathcal{O}_{\mathbb{P}^{n}}(d)\right) \rightarrow H^{0}\left(C, \mathcal{O}_{C}(d)\right)\right) .
$$

Since $C$ is 2-regular, it follows that $\alpha$ is surjective. Furthermore we have

$$
h^{0}\left(C, \mathcal{O}_{C}(d)\right)=2 d+1+h^{1}\left(C, \mathcal{O}_{C}(d)\right)=2 d+1 .
$$

Therefore we have

$$
\operatorname{codim}\left(p_{R}^{-1}(C), C \times H_{d}\right)=2 d+1
$$

for all $C \in R_{2}\left(\mathbb{P}^{n}\right)$. Thus $J_{d}$ is irreducible and

$$
\operatorname{codim}\left(J_{d}, R_{2}\left(\mathbb{P}^{n}\right) \times H_{d}\right)=2 d+1 .
$$

Further $p_{R}: J_{d} \rightarrow R_{2}\left(\mathbb{P}^{n}\right)$ is smooth by Hartshorne [3, III, Ex.10.2]. Therefore $J_{d}$ is nonsingular.

Assume that $2 d \leq 3 n-2$. Let $C \subset \mathbb{P}^{n}$ be a conic. One can choose coordinates $\left(x_{i}\right)$ on $\mathbb{P}^{n}$ such that

$$
C \subset \mathbb{P}^{2}=\left\langle x_{3}=x_{4}=\cdots=x_{n}=0\right\rangle \subset \mathbb{P}^{n}
$$


and hence

$$
C=\left\langle Q\left(x_{0}, x_{1}, x_{2}\right)=0, x_{3}=x_{4}=\cdots=x_{n}=0\right\rangle,
$$

where $Q\left(x_{0}, x_{1}, x_{2}\right)$ is a degree 2 polynomial of $x_{0}, x_{1}, x_{2}$. If a hypersurface $X \subset \mathbb{P}^{n}$ of degree $d$ contains $C$, then the equation of $X$ can be written as

$$
Q f+\sum_{i=3}^{n} x_{i} f_{i}=0
$$

where $\operatorname{deg} f=d-2, \operatorname{deg} f_{i}=d-1$. Here and afterward, $f$ and $f_{i}$ are considered as functions on $C$.

Lemma 2.2 (Notation as above). (a) The hypersurface $X$ is singular at $p \in C$ if and only if

$$
f(p)=f_{3}(p)=\cdots=f_{n}(p)=0 .
$$

(b) If $X$ is smooth along the conic $C$, then the projection $p_{H}: J_{d} \rightarrow H_{d}$ is smooth at $(C, X)$ if and only if

$$
H^{0}\left(C, \mathcal{O}_{C}(d)\right)=f H^{0}\left(C, \mathcal{O}_{C}(2)\right)+\sum_{i=3}^{n} f_{i} H^{0}\left(C, \mathcal{O}_{C}(1)\right)
$$

Proof. For $i=0,1,2$,

$$
\begin{aligned}
\left.\frac{\partial X}{\partial x_{i}}\right|_{p} & =\frac{\partial Q}{\partial x_{i}}(p) f(p)+Q(p) \frac{\partial f}{\partial x_{i}}(p)+x_{3}(p) \frac{\partial f_{3}}{\partial x_{i}}(p)+\cdots+x_{n}(p) \frac{\partial f_{n}}{\partial x_{i}}(p) \\
& =\frac{\partial Q}{\partial x_{i}}(p) f(p)=0 .
\end{aligned}
$$

However $C$ is smooth at $p$. Hence not all $\frac{\partial Q}{\partial x_{0}}(p), \frac{\partial Q}{\partial x_{1}}(p), \frac{\partial Q}{\partial x_{2}}(p)$ are zero. Therefore $f(p)=0$. For $i=3, \ldots, n$,

$$
\begin{aligned}
\left.\frac{\partial X}{\partial x_{i}}\right|_{p} & =Q(p) \frac{\partial f}{\partial x_{i}}(p)+x_{3} \frac{\partial f_{3}}{\partial x_{i}}(p)+\cdots+f_{i}(p)+x_{i} \frac{\partial f_{i}}{\partial x_{i}}(p)+\cdots+x_{n} \frac{\partial f_{n}}{\partial x_{i}}(p) \\
& =f_{i}(p)=0 .
\end{aligned}
$$

Therefore we get the first result.

Assume that $X$ is smooth along $C$. The projection $p_{H}$ is smooth at $(C, X)$ if and only if $\operatorname{ker} d p_{H}(C, X)$ has the expected dimension at $(C, X)$, i.e., $d p_{H}$ is surjective at $(C, X)$. However $R_{2}(X) \cong p_{H}^{-1}(X)$. Hence $\operatorname{ker} d p_{H}(C, X)$ is the Zariski tangent space to $R_{2}(X)$ at $C$, i.e.,

$$
\operatorname{ker} d p_{H}(C, X) \cong H^{0}\left(C, \mathcal{N}_{C / X}\right) .
$$

Hence $p_{H}$ is smooth at $(C, X)$ if and only if

$$
h^{0}\left(C, \mathcal{N}_{C / X}\right)=\operatorname{dim} J_{d}-\operatorname{dim} H_{d}=3 n-2 d-2 .
$$


Consider the exact sequence:

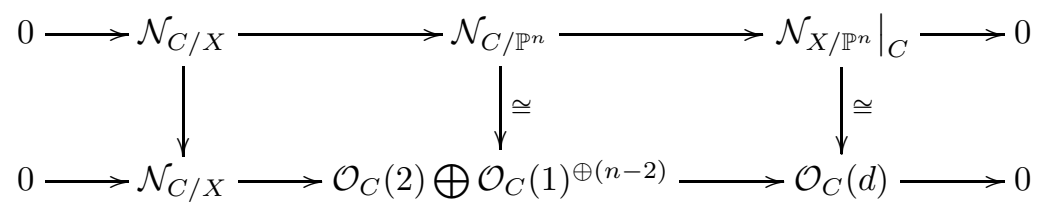

It follows that

$$
h^{0}\left(C, \mathcal{N}_{C / X}\right)=3 n-2 d-2+h^{1}\left(C, \mathcal{N}_{C / X}\right) .
$$

Therefore $p_{H}$ is smooth at $(C, X)$ if and only if $H^{1}\left(C, \mathcal{N}_{C / X}\right)=0$, which means the restriction map

$$
H^{0}\left(C, \mathcal{O}_{C}(2) \oplus \mathcal{O}_{C}(1)^{\oplus(n-2)}\right) \rightarrow H^{0}\left(C, \mathcal{O}_{C}(d)\right)
$$

is surjective. Therefore the projection $p_{H}: J_{d} \rightarrow H_{d}$ is smooth at $(C, X)$ if and only if

$$
H^{0}\left(C, \mathcal{O}_{C}(d)\right)=f H^{0}\left(C, \mathcal{O}_{C}(2)\right)+\sum_{i=3}^{n} f_{i} H^{0}\left(C, \mathcal{O}_{C}(1)\right)
$$

Fix an isomorphism $\varphi: C \rightarrow \mathbb{P}^{1}$. Then we have

$$
H^{0}\left(C, \mathcal{O}_{C}(k)\right) \cong H^{0}\left(\mathbb{P}^{1}, \mathcal{O}_{\mathbb{P}^{1}}(2 k)\right)
$$

Let

$$
\begin{aligned}
& m_{1}: H^{0}\left(\mathbb{P}^{1}, \mathcal{O}_{\mathbb{P}^{1}}(2)\right) \times H^{0}\left(\mathbb{P}^{1}, \mathcal{O}_{\mathbb{P}^{1}}(2 d-2)\right) \rightarrow H^{0}\left(\mathbb{P}^{1}, \mathcal{O}_{\mathbb{P}^{1}}(2 d)\right), \\
& m_{2}: H^{0}\left(\mathbb{P}^{1}, \mathcal{O}_{\mathbb{P}^{1}}(4)\right) \times H^{0}\left(\mathbb{P}^{1}, \mathcal{O}_{\mathbb{P}^{1}}(2 d-4)\right) \rightarrow H^{0}\left(\mathbb{P}^{1}, \mathcal{O}_{\mathbb{P}^{1}}(2 d)\right)
\end{aligned}
$$

be the multiplication maps. If $V \subset H^{0}\left(\mathbb{P}^{1}, \mathcal{O}_{\mathbb{P}^{1}}(2 d)\right)$ is a subspace, then set

$$
\begin{aligned}
& m_{1}^{-1}(V)=\left\{g \in H^{0}\left(\mathbb{P}^{1}, \mathcal{O}_{\mathbb{P}^{1}}(2 d-2)\right): m\left(H^{0}\left(\mathbb{P}^{1}, \mathcal{O}_{\mathbb{P}^{1}}(2)\right) \times\{g\}\right) \subset V\right\}, \\
& m_{2}^{-1}(V)=\left\{g \in H^{0}\left(\mathbb{P}^{1}, \mathcal{O}_{\mathbb{P}^{1}}(2 d-4)\right): m\left(H^{0}\left(\mathbb{P}^{1}, \mathcal{O}_{\mathbb{P}^{1}}(4)\right) \times\{g\}\right) \subset V\right\} .
\end{aligned}
$$

Lemma 2.3. Let $V \subset H^{0}\left(\mathbb{P}^{1}, \mathcal{O}_{\mathbb{P}^{1}}(2 d)\right)$ be a hyperplane. Then either

(a) $V=H^{0}\left(\mathbb{P}^{1}, \mathcal{O}_{\mathbb{P}^{1}}(2 d)(-p)\right)$ for some $p \in \mathbb{P}^{1}$ and

$$
m_{i}^{-1}(V)=H^{0}\left(\mathbb{P}^{1}, \mathcal{O}_{\mathbb{P}^{1}}(2 d-2 i)(-p)\right)
$$

for each $i$; or

(b) there is no such $p$, and $m_{1}^{-1}(V) \subset H^{0}\left(\mathbb{P}^{1}, \mathcal{O}_{\mathbb{P}^{1}}(2 d-2)\right)$ has codimension 3 and $m_{2}^{-1}(V) \subset H^{0}\left(\mathbb{P}^{1}, \mathcal{O}_{\mathbb{P}^{1}}(2 d-4)\right)$ has codimension 5 .

Proof. We identify $H^{0}\left(\mathbb{P}^{1}, \mathcal{O}_{\mathbb{P}^{1}}(k)\right)$ with the vector space of polynomials of degree $k$. Let $\sum_{i=0}^{2 d} u_{i} x^{i}$ denote a general polynomial of degree $2 d$ and $\sum_{i=0}^{2 d-2} v_{i} x^{i}$ 
a general polynomial of degree $2 d-2$. If $V$ is given by a linear equation $\sum_{i=0}^{2 d} c_{i} u_{i}=0$, then $m_{1}^{-1}(V)$ is given by the three equations

$$
\sum_{i=0}^{2 d-2} c_{i} v_{i}=0, \quad \sum_{i=0}^{2 d-2} c_{i+1} v_{i}=0, \quad \sum_{i=0}^{2 d-2} c_{i+2} v_{i}=0 .
$$

If these three equations are linearly dependent, then there is a point $p=(s, t) \in$ $\mathbb{P}^{1}$ such that $s c_{i}=t c_{i+1}$ for $0 \leq i \leq 2 d-2$. Equivalently,

$$
\left(c_{0}, \ldots, c_{d}\right)=\text { constant } \cdot\left(t^{d}, t^{d-1} s, \ldots, s^{d}\right) .
$$

This means that $V=H^{0}\left(\mathbb{P}^{1}, \mathcal{O}_{\mathbb{P}^{1}}(2 d)(-p)\right)$ for some $p \in \mathbb{P}^{1}$. Otherwise $m_{1}^{-1}(V)$ has codimension 3 . With similar method we can prove the other statement for $m_{2}^{-1}(V)$.

Proposition 2.4. Let $J_{d}^{0} \subset J_{d}$ be the open subset consisting of those pairs $(C, X)$ such that $X$ is smooth along $C$. Let $Z^{0} \subset J_{d}^{0}$ be the closed subset of those pairs $(C, X)$ such that $p_{H}$ is not smooth at $(C, X)$. Then we have

$$
\operatorname{codim}\left(Z^{0}, J_{d}^{0}\right) \geq 3 n-2 d-1 \text {. }
$$

Proof. It is enough to show that

$$
\operatorname{codim}\left(Z^{0} \cap p_{R}^{-1}(C), p_{R}^{-1}(C)\right) \geq 3 n-2 d-1 .
$$

Note that

$$
\begin{gathered}
J_{d}^{0} \cap p_{R}^{-1}(C) \cong\left\{\left(f, f_{3}, \ldots, f_{n}\right): f, f_{3}, \cdots, f_{n} \text { have no common zero }\right\} \\
Z^{0} \cap p_{R}^{-1}(C) \cong\left\{\left(f, f_{3}, \ldots, f_{n}\right):\right. \\
f H^{0}(C, \mathcal{O}(2))+\sum_{i=3}^{n} f_{i} H^{0}(C, \mathcal{O}(1)) \\
\left.\varsubsetneqq H^{0}(C, \mathcal{O}(d))\right\}
\end{gathered}
$$

Let $V$ be a hyperplane contained in $H^{0}\left(C, \mathcal{O}_{C}(d)\right)$. Define

$$
Z_{V}^{0}=\left\{\left(f, f_{3}, \ldots, f_{n}\right): f H^{0}\left(C, \mathcal{O}_{C}(2)\right)+\sum_{i=3}^{n} f_{i} H^{0}(C, \mathcal{O}(1)) \subset V\right\}
$$

in $H^{0}\left(C, \mathcal{O}_{C}(d-2)\right) \oplus H^{0}\left(C, \mathcal{O}_{C}(d-1)\right)^{\oplus(n-2)}$. Then we have

$$
Z^{0} \cap p_{R}^{-1}(C)=\bigcup_{V} Z_{V}^{0}
$$

Since $\operatorname{dim} \mathbb{P} H^{0}(C, \mathcal{O}(d))=2 d$, it follows that

$$
\operatorname{dim}\left(Z^{0} \cap p_{R}^{-1}(C)\right)-\operatorname{dim} Z_{V}^{0}=2 d .
$$

However we have

$$
\operatorname{dim}\left(J_{d}^{0} \cap p_{R}^{-1}(C)\right)=\operatorname{dim} p_{R}^{-1}(C) ;
$$

hence it is enough to show that

$$
\operatorname{codim}\left(Z_{V}^{0}, J_{d}^{0} \cap p_{R}^{-1}(C)\right) \geq 3 n-1 .
$$


Note that

$$
\begin{aligned}
Z_{V}^{0}= & \left\{f \in H^{0}\left(C, \mathcal{O}_{C}(d-2)\right): f H^{0}\left(C, \mathcal{O}_{C}(2)\right) \subset V\right\} \\
& \bigoplus \bigoplus_{i=3}^{n}\left\{f_{i} \in H^{0}\left(C, \mathcal{O}_{C}(d-1)\right): f_{i} H^{0}\left(C, \mathcal{O}_{C}(1)\right) \subset V\right\} \\
\cong & m_{2}^{-1}(V) \oplus m_{1}^{-1}(V)^{\oplus(n-2)} .
\end{aligned}
$$

Therefore it follows by Lemma 2.3 that

$$
\operatorname{codim}\left(Z_{V}^{0}, J_{d}^{0} \cap p_{R}^{-1}(C)\right) \geq 5+3(n-2)=3 n-1 .
$$

We are ready to prove Theorem 1.1.

Proof of Theorem 1.1. If $2 d>3 n-2$, then we have

$$
\operatorname{dim} J_{d}<\operatorname{dim} H_{d}
$$

by Lemma 2.1; hence it follows that

$$
\operatorname{codim}\left(p_{H}\left(J_{d}\right), H_{d}\right) \geq 1
$$

Therefore $R_{2}(X)$ is empty for general $X$.

Suppose that $2 d \leq 3 n-2$. Then it is not difficult to show that the map $p_{H}: J_{d} \rightarrow H_{d}$ is dominant by counting parameters: A conic $C$ in $\mathbb{P}^{n}$ is given by $n+1$ homogeneous forms $\alpha_{i}(u, v)(i=0, \ldots, n)$ of degree 2 in two variables. Taking into account the ambiguity arising from the $\mathbb{P} G L_{2}$ action of $\mathbb{P}^{1}$, a conic $C$ depends on $3 n-1$ parameters. If a conic $C$ is contained in a hypersurface $X$ in $\mathbb{P}^{n}$ defined by a homogeneous polynomial $h\left(x_{0}, \ldots, x_{n}\right)$ of degree $d$, then

$$
h\left(\alpha_{0}(u, v), \ldots, \alpha_{n}(u, v)\right)=0 .
$$

The left hand side of the above equation is a polynomial of degree $2 d$ in $u$ and $v$. Its $2 d+1$ coefficients are constantly zero. Therefore if $X$ is general, then these equations may impose independent conditions to the $3 n-1$ parameters of the conic $C$. Hence the dimension of the solutions is $3 n-2 d-2$. That is, a general hypersurface of degree $d$ in $\mathbb{P}^{n}$ contains a smooth conic. Therefore the map $p_{H}$ is dominant as asserted.

Let $H_{d}^{0} \subset H_{d}$ be the open subset parameterizing smooth hypersurfaces. If $X \in H^{0}$, then $p_{H}^{-1}(X)$ is smooth if and only if $x \notin p_{H}\left(Z^{0}\right)$. By Proposition 2.4 the codimension of $Z^{0}$ is greater than the generic fiber dimension of $p_{H}$. This shows that $R_{2}(X)$ is smooth for general $X$.

Acknowledgements. The author was supported by the research fund of Chungnam National University in 2011. 


\section{References}

[1] W. Barth and A. Vande Ven, Fano varieties of lines on hypersurfaces, Arch. Math. (Basel) 31 (1978/79), no. 1, 96-104.

[2] J. Harris, M. Roth, and J. Starr, Rational curves on hypersurfaces of low degree, J. Reine Angew. Math. 571 (2004), 73-106.

[3] R. Hartshorne, Algebraic Geometry, Graduate Texts in Mathematics, no. 52, SpringerVerlag, 1977.

[4] S. Katz, On the finiteness of rational curves on quintic threefolds, Compositio Math. 60 (1986), no. 2, 151-162.

[5] J. Kollár, Rational curves on algebraic varieties, Ergebnisse der Mathematik und ihrer Grenzgebiete. 3. Folge. A Series of Modern Surveys in Mathematics, vol. 32, SpringerVerlag, 1996.

Department of Mathematics

Chungnam National University

DAEJEON 305-764, KoreA

AND

SCHOOL OF MATHEMATICS

Korea Institute for Advanced Study

SEOUl 130-722, KoreA

E-mail address: dsshin@cnu.ac.kr 